\title{
Optimization and evaluation of an influenza A (H5) pseudotyped lentiviral particle-based serological assay
}

\author{
Jean-Michel Garcia ${ }^{\mathrm{a}, *}$, Nadège Lagarde ${ }^{\mathrm{a}}$, Edward S.K. Ma ${ }^{\mathrm{b}}$, Menno D. de Jong ${ }^{\mathrm{c}}$, J.S. Malik Peiris ${ }^{\mathrm{a}, \mathrm{b}, *}$ \\ ${ }^{a}$ HKU-Pasteur Research Centre, Hong Kong, SAR China \\ b University of Hong Kong, Hong Kong, SAR China \\ c Oxford University Clinical Research Unit, Hospital for Tropical Diseases, Ho Chi Minh City, Viet Nam
}

\section{A R T I C L E I N F O}

\section{Article history:}

Received 3 June 2009

Received in revised form 1 October 2009

Accepted 7 October 2009

\section{Keywords:}

Influenza

Pseudotyped lentiviral particles

H5N1

Diagnosis

Serology

Pseudoparticle

\begin{abstract}
A B S T R A C T
Background: Novel serological methods provide alternative options for sero-diagnosis, sero-epidemiology and for determining evidence of naturally acquired or vaccine induced immunity. Micro-neutralization tests are currently the gold standard for serological studies of highly pathogenic avian influenza in mammalian species but require handling live virus in a biosafety level (BSL) 3 environment. We previously reported the use of $\mathrm{H} 5$ pseudotyped lentiviral particles (H5pp) as an alternative to micro-neutralization tests in a BSL-2 setting (Nefkens et al., 2007).

Objective: To optimize and evaluate this newly developed H5pp assay on relevant clinical specimens.

Study design: We optimise and evaluate the performance of the H5pp assay using well-characterized sera from humans with confirmed H5N1 disease or controls.

Results: The H5pp assay is a reliable serological method for the detection and quantification of neutralizing antibody to H5-viruses.

Conclusion: H5pp provide a reliable and safe alternative for sero-diagnosis and sero-epidemiology of H5N1 infections in a BSL-2 setting.
\end{abstract}

(c) 2009 Published by Elsevier B.V.

\section{Background}

Assessment of the sero-prevalence to potentially pandemic highly pathogenic avian influenza (HPAI) H5N1 viruses is important for evaluation of its potential to transmit to humans. ${ }^{1}$ Such tests need to be reliable, safe and amenable to high throughput implementation. At present, the haemagglutination inhibition (HI) and the micro-neutralization (MN) tests are available for this purpose. ${ }^{2}$ The HI test is useful in serological studies of mammalian viruses in mammalian species or of avian viruses infecting avian species. However, the conventional HI test is not sufficiently sensitive or specific for detecting antibody responses of humans infected with

Abbreviations: HPAI, highly pathogenic avian influenza; MN, microneutralization; BSL, biosafety level; H5, haemagglutinin of H5N1 HPAI virus; H5pp, H5 pseudotyped viral particles; HI, haemagglutination inhibition assay; RTPCR, reverse transcription polymerase chain reaction; HA, haemagglutinin; MDCK, Madin-Darby canine kidney; DMEM, Dulbecco modified Eagle medium; RLU, relative luminescence unit; ROC curve, receiver operating characteristic curve; CI, confidence interval; AUC, area under curve; SE, standard error; " $c$ ", closest-to- $(0,1)$ criterion; “J", Youden index; PPV, positive predictive value; NPV, negative predictive value; NA, neuraminidase.

* Corresponding authors at: HKU-Pasteur Research Centre, Dexter H.C. Man Building, 8 Sassoon Road, Pokfulam, Hong Kong, SAR China. Tel.: +852 2816 8417; fax: +85228725782

E-mail addresses: jmgarcia@hku.hk (J.-M. Garcia), malik@hku.hk (J.S.M. Peiris). avian influenza viruses although an adaptation using horse erythrocytes does make the test more useful. ${ }^{3,4} \mathrm{MN}$, which remains the "gold standard" serological test for HPAI H5N1 at present, requires access to the live virus and to biosafety level (BSL)-3 facilities. While reverse genetics can be used to generate H5-viruses that are safe enough to be used in BSL-2 conditions, such viruses require intensive safety evaluation for non-pathogenicity in chickens and ferrets before release for use in a BSL-2 setting. ${ }^{5}$ This limits its widespread applicability of MN tests for those without access to BSL-3 facilities. Recently we introduced a BSL-2 H5 haemagglutinin pseudotyped lentiviral particle (H5pp) based assay. ${ }^{6}$ These pseudoparticles can be produced using synthetic genes based on virus haemagglutinin (HA) sequence data, even when there is no access to live virus or viral cDNA. Pseudotyping with HA only allows us to detect the functional neutralizing antibody response to the H5 without contribution from cross-reacting neuraminidase antisera. While others have also evaluated H5pp serological assays, they have not described an analysis of the parameters that affect assay performance. ${ }^{7,8}$ Moreover, their test validation has been based on small numbers of sera.

\section{Objectives}

Here we optimise H5pp assay, assess its reproducibility and define its performance characteristics using sera from patients with virologically confirmed H5N1 disease. 


\section{Study design}

\subsection{Serum samples}

Controls sera were from healthy Hong Kong residents (106 children, 102 adults and 118 persons $>60$ years of age) with no known exposure to H5N1 influenza. The "positive" test panel was 41 sera from 10 patients with RT-PCR confirmed H5N1 disease collected during the outbreak in 2003/2004 in South Vietnam from days 7 to 784 after disease onset at the Hospital for Tropical Disease of Ho Chi Minh City, Vietnam. The individual clinical details of these patients have been previously described in references. ${ }^{9-11}$ Furthermore, Supplementary Material Table S1 gives correspondence of patient ID number within the cited references to the sera tested in this evaluation. A pool of avian influenza H5 vaccinated chicken sera (MN titre 640) was used to optimize viral input and assess the reproducibility of the assay. A set of 15 subtyping polyclonal hyperimmunized chicken antisera provided by Office International des Epizooties (OIE) Reference Laboratory (Veterinary Laboratory Agency, Surrey, UK) was used for testing subtype specificity.

\subsection{H5pp assay}

H5pp were produced as described previously using codon optimized H5 gene from A/Cambodia/408008/05 H5N1. ${ }^{6}$ Two-fold serial dilutions of heat inactivated $\left(56^{\circ} \mathrm{C}, 30 \mathrm{~min}\right)$ serum were mixed with an equal volume of H5pp in culture medium (Dulbecco modified Eagle medium (DMEM) supplemented with $2.5 \%$ foetal bovine serum (Invitrogen) $1 \%$ penicillin-streptomycin (Invitrogen)) corresponding to the dose that generates $10^{5} \mathrm{RLU}$ (or otherwise mentioned) in standardized conditions, incubated for $2 \mathrm{~h}$ at $37^{\circ} \mathrm{C}$ and then added to a preformed monolayer of Madin-Darby canine kidney (MDCK) cells. After $48 \mathrm{~h}$ incubation, luminescence was read after addition of Steady-Glo (Promega) luciferase substrate with either Micro-beta (PerkinElmer) or Glomax (Promega) plate readers. The neutralization of infection was detected by measuring the reduction of end-point signal versus controls done in absence of serum or in absence of virus, regarded as equivalent to $0 \%$ or $100 \%$ neutralization, respectively.

\subsection{Micro-neutralization test (MN)}

The micro-neutralization test was done as described previously. ${ }^{12}$ One hundred tissue culture infectious dose 50 $\left(100 \mathrm{TCID}_{50}\right)$ of A/Vietnam/1194/04 (H5N1) virus was mixed with an equal volume of 2 -fold serial dilutions of serum in quadruplicate, incubated for $1 \mathrm{~h}$ at $37^{\circ} \mathrm{C}$ and the virus antibody mixture was added to a preformed monolayer of MDCK cells. The plates were incubated for 3 days and the cytopathic effect read under an inverted microscope. Virus back titrations were included to confirm whether the challenge dose was as expected.

\subsection{Determination of neutralizing titer}

In MN assay, titer was defined by the reciprocal highest serum dilution protecting $\geq 50 \%$ of the wells. In H5pp assay, the reciprocal dilution giving 50\% (unless otherwise specified) neutralization was computed from the neutralization curved fitted with the Hill equation. ${ }^{13}$ This takes into consideration information from all dilutions and does not overly rely on few quadruplicate serum dilutions to determine the titer for a $50 \%$ effect as is done using the Reed and Muench method. ${ }^{14}$

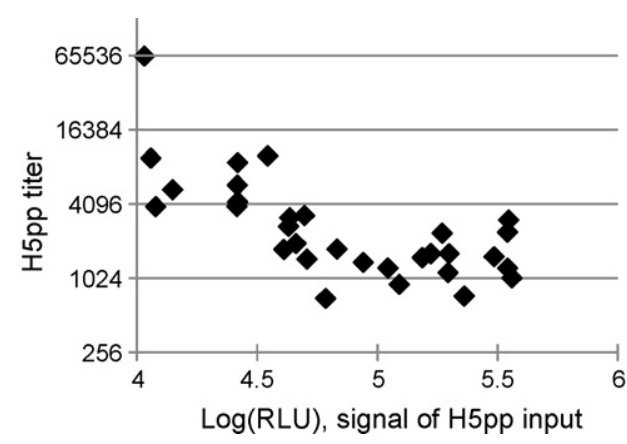

Fig. 1. Optimization of virus dose in $\mathrm{H} 5$ pseudotype assay. Neutralization titers plotted against the input of H5pp expressed as log value of signal in relative luminescence unit (RLU) measured in absence of sera. Values represent the mean of duplicates.

\subsection{Determination of diagnostic parameters}

A detailed description of the computation and interpretation these parameters (sensitivity, specificity, positive or negative predictive values (PPV or NPV) and accuracy) can be found in references. ${ }^{15,16}$

\subsection{Statistical analysis}

Bland-Altman analysis was used to evaluate the systematic bias and limits of agreement of H5pp versus MN. ${ }^{17}$ The logtransformation of titers was required for a normal distribution, an assumption of the statistical test used, verified using normal (quantile-quantile) plot (data not show). All calculations were done in Excel $^{\mathrm{TM}}$.

\section{Results}

\subsection{Determination of optimal amount of H5pp}

In 33 independent experiments, we measured the titer of a single pooled chicken serum for different batches of H5pp (mean values of duplicate were used for the calculations). We found that once the H5pp input exceeds around $10^{5} \mathrm{RLU}$, the titer is relatively independent of the H5pp concentration used (Fig. 1).

\subsection{Determination of optimal cut-offs}

The receiver operating characteristic (ROC) curve was used for determination of optimal cut-off point. ${ }^{18}$ It was generated by plotting the sensitivity as a function of 1-specificity (Fig. 2A). ${ }^{19}$ The optimal diagnostic effectiveness was determined using the sensitivity/specificity that gives either the minimal value for $(1-\text { sensitivity })^{2}+(1-\text { specificity })^{2}$, called the "closest-to- $(0,1)$ " criterion, denoted " $c$ "; or the maximal value for (sensitivity + specificity - 1), the Youden Index, ${ }^{20}$ denoted " J". We computed ROC curves, " $c$ " and " $J$ " criteria using the panel of human sera by simultaneously varying the positivity threshold (range [10-640]) and the percentage of neutralization (range [5-95\%]) used to define the titer. Both criteria identify the same optimal cut-off values corresponding to 50\% neutralization (Fig. 2B) and to positivity threshold of a serum dilution of $\geq 20$ (Fig. 3). Furthermore, $50 \%$ signal reduction is the value for which fitting using the Hill equation is the most robust (inflection point). These ROC curves also indicate that the assay has excellent accuracy as estimated by the area under curve (AUC $>0.99$ ) with a corresponding standard errors (SE) of $<0.5 \%{ }^{21}$ 

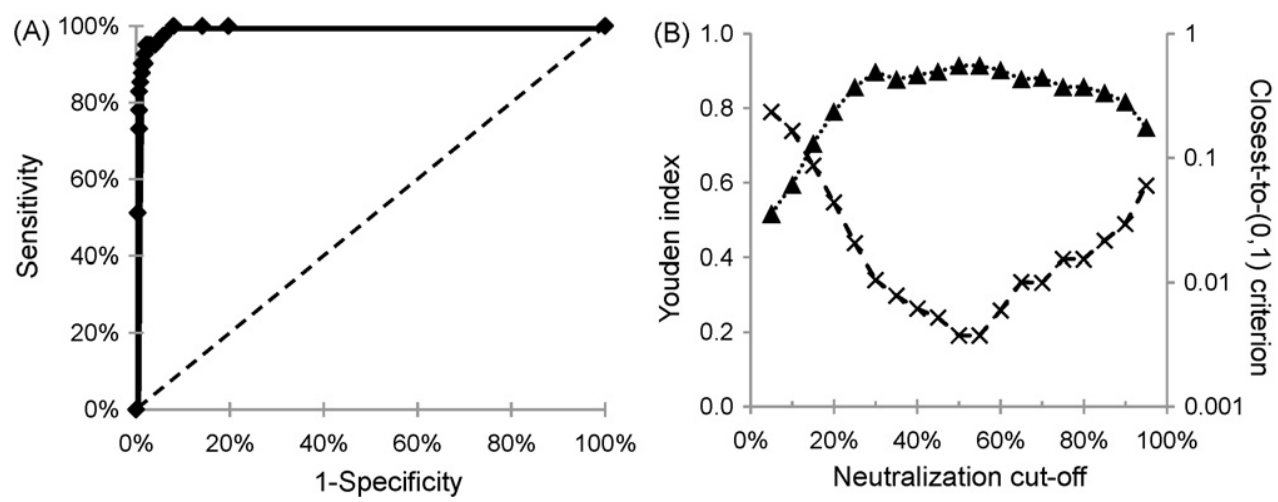

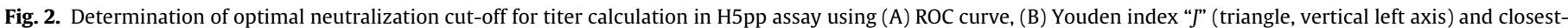
to- $(0,1)$ "c" criterion (cross, vertical right axis).
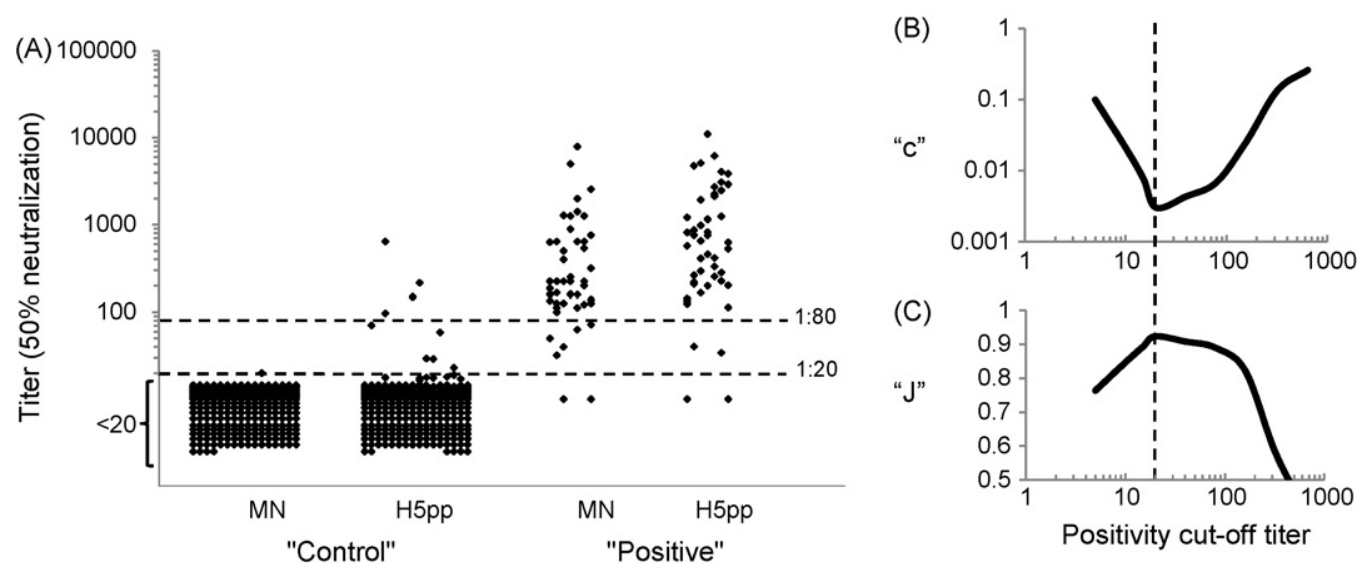

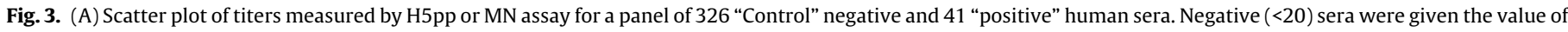

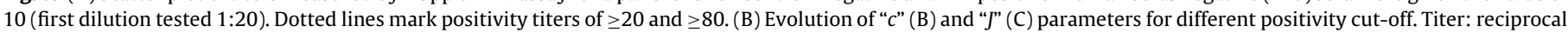
dilution giving $50 \%$ neutralization.

\subsection{Assay reproducibility}

The pooled chicken serum and a single batch of H5pp were used to assess the reproducibility of the assay over 3 batches (tested on 3 different weeks) of 3 plates, each containing 6 replicates. Coefficients of variation ( $C V \%$ ) were $\mathrm{CV}_{\text {intra-plate }}<5 \%, \mathrm{CV}_{\text {inter-plate }}<3 \%$ and $\mathrm{CV}_{\text {inter-batch }}<7 \%{ }^{22}$ All variances calculated were homogenous according to Cochran test at the level of significance $\alpha=0.05$.

\subsection{Agreement of titers measured with H5pp or MN tests in human sera}

Titers measured with H5pp are higher than with the MN test (Fig. 4A) suggesting a higher sensitivity in detecting H5 antibody. Using the statistical method developed by Bland and Altman, ${ }^{17}$ we found that $\mathrm{H} 5 \mathrm{pp}$ titers exceed the MN titers on average by 2 -fold (95\% CI: $1.5-2.5)(p=0.002)$.
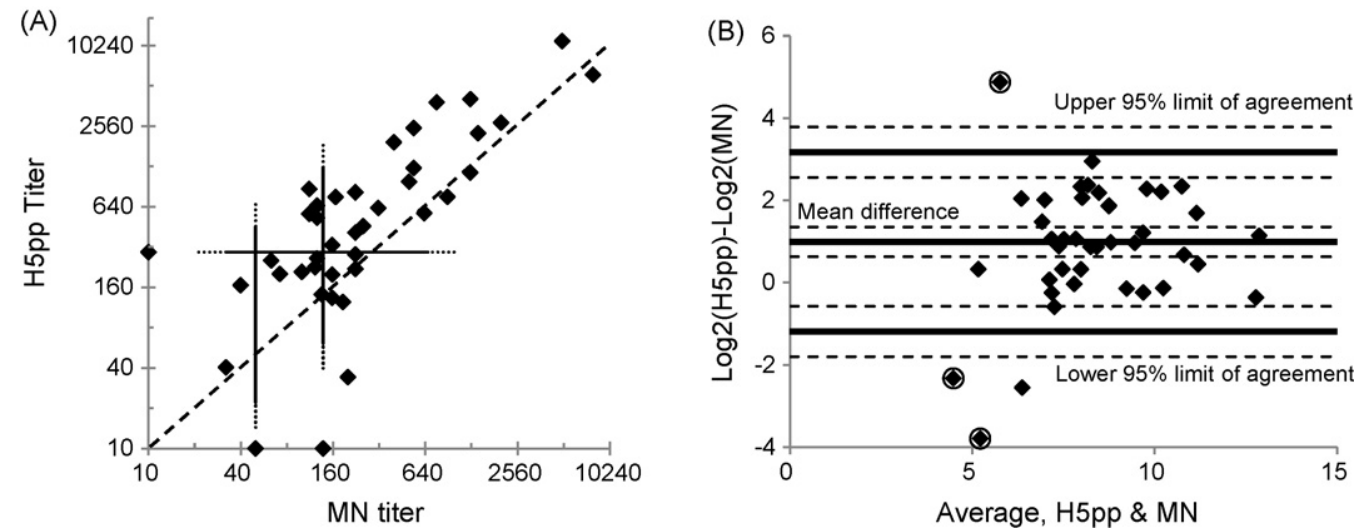

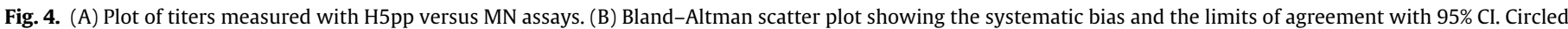

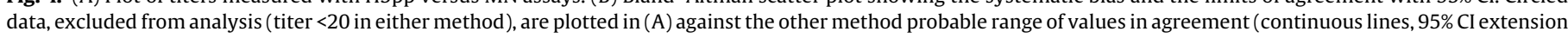
in dotted line). 
Table 1

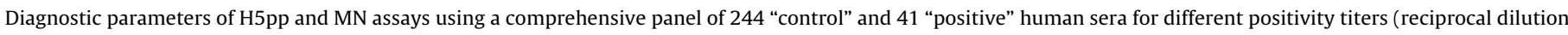

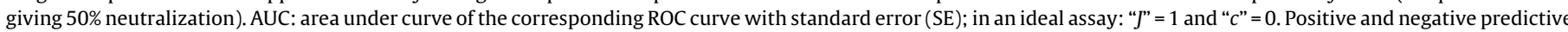
values are noted PPV and NPV, respectively.

\begin{tabular}{|c|c|c|c|c|c|c|c|c|c|}
\hline \multirow[t]{2}{*}{ Positivity cut-off } & \multicolumn{3}{|l|}{ H5pp assay } & \multicolumn{3}{|c|}{ MN assay } & \multicolumn{3}{|c|}{$\begin{array}{l}\text { Screening with H5pp and confirming } \\
\text { those with H5pp titers } \geq 20 \text { by MN test }\end{array}$} \\
\hline & $\geq 80$ & $\geq 40$ & $\geq 20$ & $\geq 80$ & $\geq 40$ & $\geq 20$ & $\geq 80^{\mathrm{a}}$ & $\geq 40^{\mathrm{a}}$ & $\geq 20^{\mathrm{a}}$ \\
\hline Sensitivity & $90 \%$ & $93 \%$ & $95 \%$ & $85 \%$ & $95 \%$ & $98 \%$ & $83 \%$ & $90 \%$ & $93 \%$ \\
\hline Specificity & $99 \%$ & $98 \%$ & $97 \%$ & $100 \%$ & $100 \%$ & $100 \%$ & $100 \%$ & $100 \%$ & $100 \%$ \\
\hline PPV & $90 \%$ & $86 \%$ & $81 \%$ & $100 \%$ & $100 \%$ & $98 \%$ & $100 \%$ & $100 \%$ & $97 \%$ \\
\hline NPV & $99 \%$ & $99 \%$ & $99 \%$ & $98 \%$ & $99 \%$ & $100 \%$ & $98 \%$ & $99 \%$ & $99 \%$ \\
\hline Accuracy & $98 \%$ & $98 \%$ & $97 \%$ & $98 \%$ & $99 \%$ & $99 \%$ & $98 \%$ & $99 \%$ & $99 \%$ \\
\hline Closest-to- $(0,1), c$ & 0.010 & 0.006 & 0.003 & & & & & & \\
\hline Youden index, $J$ & 0.89 & 0.91 & 0.92 & & & & & & \\
\hline $\operatorname{AUC}(\mathrm{SE})$ & $0.994(0.36 \%)$ & $0.995(0.34 \%)$ & $0.995(0.33 \%)$ & & & & & & \\
\hline
\end{tabular}

a Positivity cut-off in confirmatory MN assay.

Table 2

Subtype specificity of H5pp using an OIE panel of reference hyper-immune chicken antisera.

\begin{tabular}{lll}
\hline Subtype & Viral strain & H5pp titer \\
\hline H1N1 & A/Turkey/England/250/79 & $<20$ \\
H2N2 & A/Singapore/1/57 & $<20$ \\
H3N2 & A/Turkey/England/69 & $<20$ \\
H4N6 & A/Duck/Czech/56 & $<20$ \\
H5N1 & A/Chicken/Scotland/59 & 1171 \\
H5N3 & A/Tern/South Africa/61 & 1469 \\
H6N8 & A/Ostrich/R.S.A./946/98 & $<20$ \\
H7N7 & A/England/268/96 & $<20$ \\
H8N4 & A/Turkey/Ontario/68 & $<20$ \\
H9N2 & A/Turkey/Wisconsin/1/66 & $<20$ \\
H10N9 & A/Egyptian goose/S. Africa/238/98 & $<20$ \\
H11N6 & A/Duck/England/56 & $<20$ \\
H12N5 & A/Duck/Alberta/60/76 & $<20$ \\
H14N6 & A/Mallard/Gurjev/244/82 & $<20$ \\
H16N3 & A/Gull/Denmark/68110/02 & $<20$ \\
\hline
\end{tabular}

\subsection{Performance characteristics of the H5pp and MN assays on human serum panels}

Using the optimized H5pp assay parameters, we compared the H5pp assay with MN at different cut-off titres for positivity (Table 1 ). At the positivity threshold $\geq 80$ defined by WHO, ${ }^{1}$ the performance of the H5pp test compares favourably with MN. At a lower cut-off titer $(\geq 20)$, the sensitivity of the H5pp test improves (from $90 \%$ to $95 \%$ ) while the specificity drops (from 99\% to $97 \%$ ).

In the 326 control sera, 317 sera were negative in both assays. Nine had H5pp titers $\geq 20$ (one also had an MN titer of 20) and seven of these were from those over 60 years of age. When a titer of $\geq 80$ was used as cut-off, 4 sera remained $\mathrm{H} 5 \mathrm{pp}$ positive (Fig. $3 \mathrm{~A}$ ). In the positive panel of 41 sera, the H5pp and MN tests yield 4 and 6 false negative sera, respectively at a cut-off of $\geq 80$; while 2 and 1 sera, respectively, remain false negative at a titer of $\geq 20$. The latter $\mathrm{MN}$ data is comparable to a previous study on naturally infected H5N1 patient. $^{23}$

\subsection{Subtype specificity}

Using the panel of hyper-immune sera to 14 influenza virus subtypes (H13 and H15 were not available), we confirmed lack of cross-reaction to non-H5 subtypes (Table 2 ).

\section{Discussion}

We optimized and evaluated a $\mathrm{H} 5$ pseudoparticle-based assay as an alternative to the existing MN method for detection of neutralizing antibodies against the potentially pandemic $\mathrm{H} 5$ avian influenza.
We established that increasing quantities of H5pp led to progressive reduction of the antibody titer until the latter reaches or exceeds around $10^{5}$ RLU equivalents per well, beyond which point the H5pp antibody titer becomes independent of the input H5pp virus. Keeping the H5pp value above $10^{5}$ RLU minimises batch-tobatch variations in the antibody titers. It should be noted that the numerical value of luminescence is dependent on the plate reader used ("relative" luminescence unit) as each machine has different signal amplification gain. Therefore, this threshold limit may have to be re-optimized if a different plate reader is used.

We assessed the H5pp assay using a test panel of 326 control sera from the general population in Hong Kong considered as true negative and 41 sera from patients with virologically confirmed H5N1 disease as true positive. An optimal positivity titer threshold of 20 was found to maximise both sensitivity and specificity. However the internationally accepted positivity cut-off titer in the $\mathrm{MN}$ assay for H5N1 is defined as 80 to minimise the frequency of false-positive results. We have therefore analysed our data at both cut-off antibody levels. Most "false-positive" sera identified in the $\mathrm{H} 5 \mathrm{pp}$ test were those over 60 years of age. It is now recognised that a proportion of elderly persons have neutralizing antibodies to H5N1 virus. ${ }^{24}$ In that regard, the current panel of sera for test evaluation is particularly stringent because $36 \%$ of the control panel are $>60$ years of age. At a positive cut-off titer of $\geq 80$, the H5pp test compares favourably with the MN test. The specificity and PPV (Table 1) of the H5pp test will be higher in those younger than 60 years of age (i.e. $99 \%$ and $97 \%$, respectively, data not shown).

Alternatively, an effective application of the H5pp test would be to use it as a high throughput screening assay at BSL-2 containment with the sera with titers $\geq 20$ being confirmed in a reference BSL3 laboratory setting with the MN titer $\geq 80$. Such a strategy leads to diagnostic performances that are not markedly inferior to that obtained by screening all the sera in BSL-3 containment using the MN test (Table 1 ). This approach has major advantages in laboratories with no access to BSL-3, including many developing countries that are in the front-line in confronting HPAI H5N1 infection.

H5pp can be made to carry the virus HA alone or to have both HA and NA. While the latter is technically easier to produce (unpublished data) we have chosen to produce pseudotyped particles that only contain the HA to have the opportunity to eliminate some potential cross-subtype reactivity that may arise between for example the NA of H5N1 and of the seasonal influenza virus H1N1. The strong positive correlation we find between titers obtained with the H5pp (only HA present) and MN (complete virus with HA and NA) tests indicates that the HA plays a dominant role in the neutralizing antibody response.

In conclusion, we have developed and evaluated a high throughput screening assay that can be carried out in BSL-2 containment. By testing a comprehensive set of sera, we have demonstrated 
that the H5pp assay faithfully replicates the results with the MN test. Therefore, H5pp assay can be a safer alternative to MN for monitoring the neutralizing antibody levels in applications such as sero-epidemiological investigation of avian influenza or H5N1 related vaccine immunity assessment.

\section{Conflict of interest}

The authors declare no conflict of interest.

\section{Acknowledgements}

This work was supported by the University Grants Committee of the Hong Kong Special Administrative Region, China (Project No AoE/M-12/06), the French Ministry of Health, RESPARI Pasteur network and the Li Ka Shing Foundation

\section{Appendix A. Supplementary data}

Supplementary data associated with this article can be found, in the online version, at doi:10.1016/j.jcv.2009.10.009.

\section{References}

1. Abdel-Ghafar AN, Chotpitayasunondh T, Gao Z, Hayden FG, Nguyen DH, de Jong $\mathrm{MD}$, et al. Writing committee of the second world health organization consultation on clinical aspects of human infection with avian influenza A (H5N1) virus. Update on avian influenza A (H5N1) virus infection in humans. $N$ Engl J Med 2001;358:261-73.

2. WHO. Manual on animal influenza diagnosis and surveillance, $\mathrm{WHO} / \mathrm{CDS} / \mathrm{CSR} / \mathrm{NCS} / 2002.5$.

3. Kayali G, Setterquist SF, Capuano AW, Myers KP, Gill JS, Gray GG. Testing human sera for antibodies against avian influenza viruses: horse RBC hemagglutination inhibition vs. microneutralisation assays. J Clin Virol 2008;43:73-8.

4. Stephenson I, Wood JM, Nicholson KG, Charlett A, Zambon MC. Detection of anti-H5 responses in human sera by HI using horse erythrocytes following MF59-adjuvanted influenza A/Duck/Singapore/97 vaccine. Virus Res 2004;103:91-5.

5. Nicolson C, Major D, Wood JM, Robertson JS. Generation of influenza vaccine viruses on Vero cells by reverse genetics: an H5N1 candidate vaccine strain produced under a quality system. Vaccine 2005;23:2943-52.
6. Nefkens I, Garcia JM, Lagarde N, Chu K, Peiris JS, Buchy P, et al. Hemagglutinin pseudotyped lentiviral particles: characterization of a new method for avian H5N1 influenza sero-diagnosis. J Clin Virol 2007;39:27-33.

7. Temperton NJ, Hoschler K, Major D, Nicolson C, Manvell R, Hien VM, et al. A sensitive retroviral pseudotype assay for influenza H5N1-neutralizing antibodies. Influenza Other Respi Viruses 2007;1:105-12.

8. Wang W, Butler EN, Veguilla V, Vassell R, Thomas JT, Moos M, et al. Establishment of retroviral pseudotypes with influenza hemagglutinins from $\mathrm{H} 1, \mathrm{H} 3$, and $\mathrm{H} 5$ subtypes for sensitive and specific detection of neutralizing antibodies. J Virol Methods 2008;153:111-9.

9. de Jong MD, Simmons CP. Thanh TT, Hien VM, Smith GJ, Chau TN, et al. Fatal outcome of human influenza A (H5N1) is associated with high viral load and hypercytokinemia. Nat Med 2006;12:1203-7.

10. de Jong MD, Tran TT, Truong HK, Vo MH, Smith GJ, Nguyen VC, et al. Oseltamivir resistance during treatment of influenza A (H5N1) infection. $N$ Engl J Med 2005;353:2667-72.

11. Tran TH, Nguyen TL, Nguyen TD, Luong TS, Pham PM, Nguyen VC, et al. Avian influenza A (H5N1) in 10 patients in Vietnam. N Engl J Med 2004;350:1179-88.

12. Choi YK, Nguyen TD, Ozaki H, Webby RJ, Puthavathana P, Buranathal C, et al. Studies of H5N1 influenza virus infection of pigs by using viruses isolated in Vietnam and Thailand in 2004. J Virol 2005;79:10821-5.

13. Hill AV. The possible effects of aggregation of the molecules of hemoglobin on its dissociation curves. J Physiol (Lond) 1910;40:4-7.

14. Reed LJ, Muench H. A simple method of estimating fifty percent endpoints. Am J Hyg 1938;27:493-7.

15. Akoben AK. Understanding diagnostic tests 1 : sensitivity, specificity and predictive values. Acta Paediatr 2006;96:338-41.

16. Douglass CW. Evaluating diagnostic tests. Adv Dent Res 1993;7:66-9.

17. Bland JM, Altman DG. Statistical methods for assessing agreement between two methods of clinical measurement. Lancet 1986;1:307-10.

18. Akoben AK. Understanding diagnostic tests 3: receiver operating characteristic curves. Acta Paediatr 2007;96:644-7.

19. Perkins NJ, Schisterman EF. The inconsistency of 'optimal' cutpoints obtained using the two criteria based on the receiver operating characteristics curve. Am J Epidemiol 2006;163:670-5.

20. Fluss R, Faraggi D, Reiser B. Estimation of the Youden Index and its associated cutoff point. Biomed J 2005;47:458-72.

21. Hanley JA, McNeil BJ. The meaning and use of the area under the receiver operating characteristic (ROC) curve. Radiology 1982;143:29-36.

22. Caporal-Gautier J, Nivet JM, Algranti P, Guilloteau M, Histe M, Lallier M, et al. Guide de validation analytique. Rapport d'une commission SFSTP. I. Methodologie. STP Pharma Pratiques 1992;2:205-26.

23. Katz JM, Lim W, Bridges CB, Rowe T, Hu-Primmer J, Lu X, et al. Antibody response in individuals infected with avian influenza $A$ (H5N1) viruses and detection of anti-H5 antibody among household and social contacts. J Infect Dis 1999;180:1763-70.

24. Treanor JJ, Campbell JD, Zangwill KM, Rowe T, Wolff M. Safety and immunogenicity of an inactivated subvirion influenza A (H5N1) vaccine. $N$ Engl J Med 2006;354:1343-51. 